\title{
Evaluación de registros holter durante 19 horas en el contexto de la teoría de la probabilidad
}

\author{
Javier Rodríguez ${ }^{1}$, Signed Prieto ${ }^{2}$, Jaime Paéz ${ }^{3}$, Catalina Correa $^{2}$, Carlos Montenegro $^{4}$, Jairo Cortés ${ }^{5}$, Mario Castro ${ }^{4}$ \\ 1 Director del Grupo Insight. Centro de Investigaciones Clínica del Country. Bogotá, Colombia. \\ 2 Investigadora Grupo Insight. Centro de Investigaciones Clínica del Country. Bogotá, Colombia. \\ 3 Ing. MSc. Director Grupo de Investigación NEOTIC. Facultad de Ingeniería. Universidad Cooperativa de Colombia. Bogotá, Colombia. \\ 4 Ing. PhD. Investigador Grupo NEOTIC Facultad de Ingeniería. Universidad Cooperativa de Colombia. Bogotá, Colombia. \\ 5 Ing. MSc. Investigador Grupo de Investigación NEOTIC. Facultad de Ingeniería. Universidad Cooperativa de Colombia. Bogotá, Colombia.
}

Introducción: Previamente se desarrolló una nueva metodología de ayuda diagnóstica para los registros Holter fundamentada en los sistemas dinámicos y la teoría de probabilidad, a partir de la información registrada en 21 horas.

Objetivo: Evaluar la capacidad diagnóstica de esta metodología durante 19 horas, comparándola con los resultados convencionales del Holter y con los resultados del método matemático aplicado en 21 horas.

Materiales y Métodos: fueron evaluados $80 \mathrm{ca}-$ sos de pacientes mayores a 20 años, 10 con registro Holter normal y 70 diagnosticados de forma convencional con diferentes patologías cardíacas. Se establecieron los rangos para las frecuencias cardíacas y de número de latidos por hora en 21 y 19 horas; luego, se calculó la probabilidad de ocurrencia de estos, lo que permitió diferenciar estados de normalidad y enfermedad aguda a partir de tres parámetros. Se comparó el diagnóstico físico-matemático con el diagnóstico convencional, tomado como Gold Standard.

Resultados: De los casos normales, dos presentaron probabilidad menor o igual a 0,217 y ocho probabilidades mayores o igual a 0,304 ; ningún caso de enfermedad aguda presentó valores con probabilidad menor o igual a 0,217 , mientras que todos presentaron valores mayores o iguales a 0,304 , tanto para los registros Holter evaluados en 21 como en 19 horas.

Conclusiones: Se confirmó la utilidad clínica de la metodología ante una reducción del tiempo de evaluación a 19 horas, obteniendo diagnósticos objetivos con base en la auto-organización matemática del fenómeno. 


\section{Evaluation of 19-hour holter recordings in the context of probability theory}

Background: a new method to help evaluate 21hour holter recordings based on dynamic systems and the theory of probability was previously developed

Aim: to evaluate the diagnostic value of this methodology in the analysis of $19 \mathrm{hr}$ compared to conventional holter analysis over a 21 -hr recording.

Methods: the holter recordings of 80 subjects aged over 20 years old were analyzed. Ten subjects had a normal holter and 70 conventionally diagnosed as abnormal. Ranges for heart rate and number of beats in 21 or 19 hours were determined. The probability of their occurrence was calculated using 3 parameters. The mathematically derived diagnosis was compared to the clinical diagnosis, considered a gold standard. Results: Among normal cases the calculated probability was $\leq 0.217$ in 2 cases and $\geq 0.304$ in 8 . No case with acute disease presented probability values $\leq 0.217$; all had probability values $\geq 0.304$, both in 21 and 19 hour recordings.

Conclusion: the mathematical methodology described was clinically useful allowing a reduction in recording time from 21 to $19 \mathrm{hr}$. Clinical diagnosis may be inferred from the mathematical organization of a holter recording.

Keywords: holter recording, heart rate, probability theory. 


\section{Introducción:}

Diferentes teorías físico-matemáticas han permitido obtener soluciones a problemas clínicos específicos. Dentro de ellas, se encuentra la teoría de los sistemas dinámi$\cos ^{1-3}$. Esta estudia el estado y evolución de los sistemas. Las representaciones geométricas de la evolución del sistema son denominadas atractores y se evidencian en un espacio matemático denominado espacio de fase 1,3; estas representaciones geométricas pueden presentar un comportamiento periódico, caótico o puntual. En oposición a las concepciones tradicionales de la fisiología $a^{4}$, se ha encontrado que, en medicina, las variables que describen la dinámica cardíaca son asociadas a atractores caóticos $^{5-7}$.

Entre el grupo de enfermedades no transmisibles se encuentran las Enfermedades Cardio Vasculares (ECV), que representan la mayor cantidad de muertes según la Organización Mundial de la salud (OMS), con una tasa de mortalidad que asciende a $30 \%$ a nivel mundial y abarcando diagnósticos como las cardiopatías y los accidentes vasculares cerebrales ${ }^{8}$.

Por medio del monitoreo Holter, es posible registrar cualquier alteración que se presente en un periodo de 24 a 48 horas o 14 días ${ }^{10}$. La complejidad de la interpretación de estos resultados obstaculiza el desarrollo de algoritmos que permitan automatizar los diagnósticos, a pesar de que existen protocolos con la finalidad guiar el dictamen clínico. Surge, entonces, la necesidad de proponer nuevas metodologías objetivas y reproducibles que solventen las limitaciones actuales del diagnóstico de las patologías cardíacas. Uno de los primeros estudios diseñados en el contexto de las dinámicas no lineales estableció una nueva concepción de salud-enfermedad, al estudiar la fisiología cardíaca por medio de la teoría de los sistemas dinámicos ${ }^{11}$, asociando un comportamiento muy estable o bien muy aleatorio a un estado patológico, mientras que la normalidad se asoció a un estado intermedio entre estos dos comportamientos. Partiendo de esta base y por medio del análisis de medidas fractales, se propusieron índices predictores de mortalidad en pacientes que han sufrido infarto agudo de miocardio (IAM) con fracción de eyección (FE) reducida, siendo éste un parámetro más confiable que la variabilidad del $\mathrm{RR}^{12}$.

La literatura médica actual ha dado a conocer trabajos de investigación desarrollados para caracterizar la dinámica cardíaca en el contexto de la teoría de los sistemas dinámicos ${ }^{13}$ y la teoría de la probabilidad ${ }^{14-16}$. Uno de estos trabajos analiza el número de rangos de aparición del número de latidos mínimos, máximos y total de latidos durante 21 horas, mediante una metodología funda-

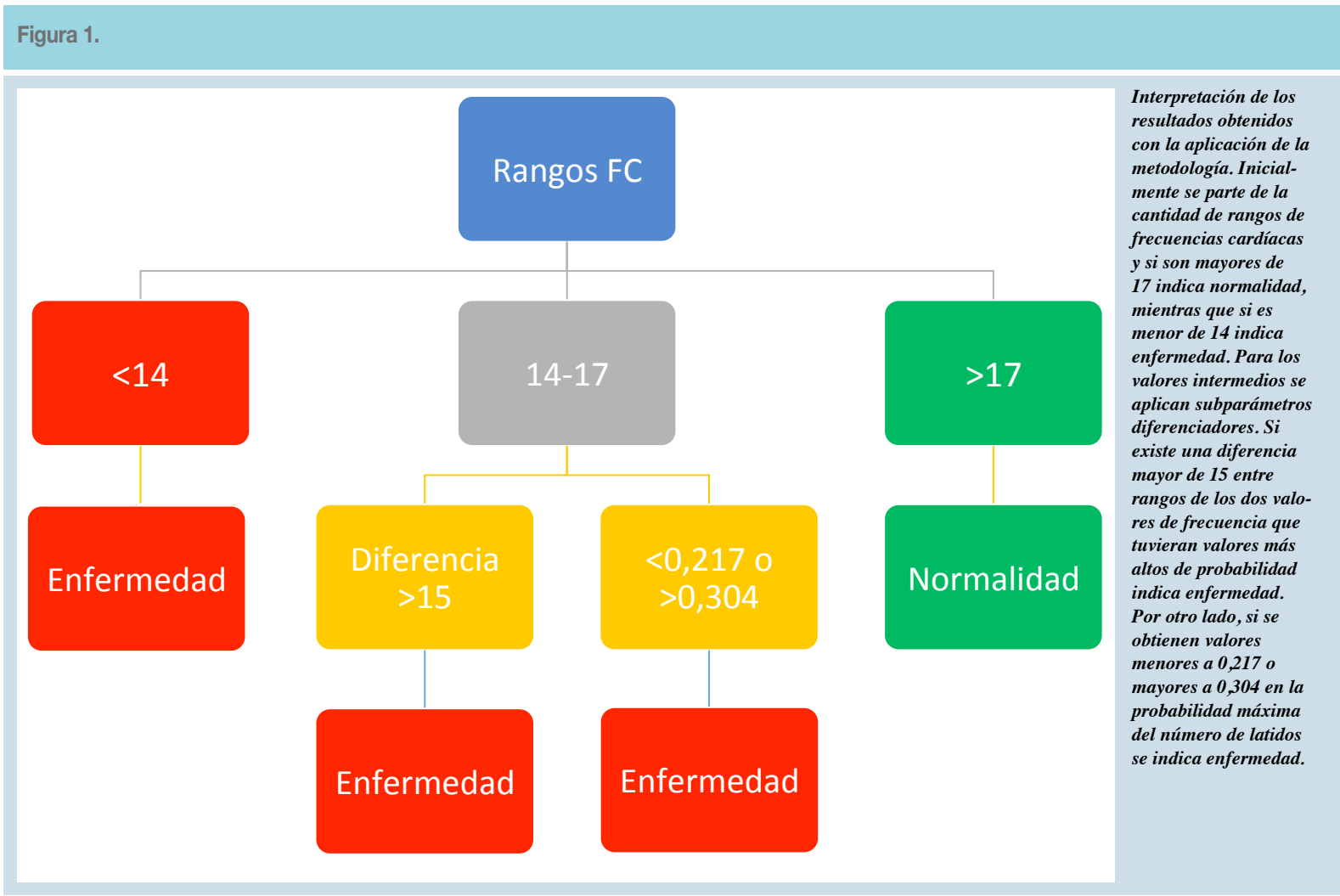


mentada en la teoría de la probabilidad. Los resultados de este estudio permitieron hacer distinciones matemáticas entre estados de normalidad y enfermedad, así como la evolución hacia alguno de estos dos estados. Posteriormente, se confirmó la reproductibilidad y aplicabilidad clínica de la metodología en un estudio ciego, hallando valores de sensibilidad y especificidad del $100 \%$ y coeficiente Kappa de 115. Adicionalmente, un estudio llevado a cabo en pacientes con y sin tratamiento de metoprolol confirmó la efectividad del método para evaluar el impacto de tratamientos farmacológicos en pacientes con diferentes tipos de arritmia cardíaca ${ }^{16}$.

El propósito de este trabajo es disminuir el tiempo de evaluación necesario para obtener un diagnóstico objetivo mediante la metodología basada en la teoría de la probabilidad y la teoría de los sistemas dinámicos ${ }^{14}$, reduciendo el registro de la dinámica cardíaca de 21 horas a 19 , con el fin de facilitar a futuro su aplicabilidad clínica.

\section{Materiales y métodos:}

\section{Definiciones}

Rango de frecuencia cardíaca: intervalo de 5 latidos/ minuto del cual se obtiene el valor de la frecuencia cardíaca.

Rango del número de latidos: rango de 250 latidos en el que se encuentra el número total de latidos de cada hora.

Probabilidad del Rango: cociente entre la frecuencia de un rango medido $\mathrm{N}$ y el total de repeticiones de los rangos medidos $\mathrm{N}$.

$$
P(A)=\frac{\text { Repeticiones del rango } \mathrm{r}}{\text { Total de repeticiones de los rangos medidos }}=\frac{N_{R}}{N}
$$

\section{Población}

Se analizaron 80 registros Holter provenientes de bases de datos del grupo Insight de individuos con edad superior a los 20 años 10 de sujetos con registro Holter evaluados como normales y 70 con un diagnóstico electrocardiográfico que indicaba alteración en el comportamiento de la dinámica cardíaca. Dichos diagnósticos fueron emitidos por un cardiólogo experto de acuerdo con los parámetros clínicos convencionales.

\section{Procedimiento}

Inicialmente, se enmascararon los diagnósticos establecidos por el experto clínico. Se tomaron los valores máximos, mínimos e intermedios de la frecuencia cardíaca du- rante 19 y 21 horas. Dichos valores fueron divididos en rangos de cinco latidos por minuto, con el fin de calcular la probabilidad del número de frecuencias obtenidas en cada rango con respecto al total de frecuencias de cada registro Holter. Posteriormente, se cuantificó el número de rangos de las frecuencias cardíacas en cada paciente, la diferencia entre los rangos de los dos valores de frecuencia con probabilidad más elevada y la sumatoria de las probabilidades de estos valores. Así mismo, se evaluó la probabilidad del número total de latidos por hora mediante rangos de 250 latidos, en 21 y 19 horas, estableciendo la máxima probabilidad evidenciada en cada registro; también, se evaluó el número de latidos mínimo y máximo. Los valores encontrados se analizaron con base en los 3 parámetros diagnósticos previamente establecidos ${ }^{14}$, y que son descritos a continuación, en aras de determinar el diagnóstico matemático de cada dinámica: Valores mayores a 17 en el número de rangos de frecuencia cardíaca (Figura 1) son indicativos de normalidad; valores menores de 14 son indicativos de enfermedad. Para establecer el diagnóstico de los valores intermedios se aplica el parámetro 2, formado por dos subparámetros que son aplicados de manera simultánea: a) si se evidencia una diferencia mayor o igual a 15 entre los rangos de los dos valores de frecuencia que tuvieron los valores más elevados de probabilidad, se establece el diagnóstico de enfermedad. b) Valores de probabilidad iguales o menores a 0,217 o mayores o iguales a 0,304 presentes en la probabilidad máxima del número de latidos indican enfermedad. Con esto se establece la evaluación, de forma tal que, si únicamente se presenta el parámetro a), se diagnostica enfermedad; si se presentan simultáneamente los parámetros a) y b), hay enfermedad; si únicamente se presenta el parámetro b, se trata de un estado evolutivo hacia la enfermedad; si se presenta el parámetro b y un número de latidos inferior a 3.000 o mayor a 6.250, hay enfermedad. Parámetro 3 , cuando se encuentran valores superiores a 0,319 en la suma de las dos probabilidades de mayor frecuencia, se establece el diagnóstico de enfermedad; también, cuando se presenta una de las siguientes opciones: los subparámetros a) y b) del parámetro 2 también presentan valores relacionados con enfermedad; el parámetro b es indicativo de enfermedad; o se registra un número mayor a 6250 latidos o inferior a 3000 en una hora, y el parámetro b se asocia a enfermedad ${ }^{14}$. Finalmente, se realizó una comparación entre los diagnósticos matemáticos obtenidos en 21 y 19 horas, con el fin de establecer similitudes y/o diferencias entre ambos. 


\section{Análisis estadístico}

Para efectos del análisis estadístico se tomó como Gold Standard el diagnóstico establecido por el cardiólogo experto. Se comparó dicho diagnóstico con los resultados establecidos por el método matemático en 19 horas, a través del cálculo de especificidad y sensibilidad. Dichas mediciones fueron establecidas a partir de una clasificación binaria: los verdaderos positivos (VP) corresponden al número de registros evaluados como patológicos mediante el método matemático y los parámetros clínicos convencionales. Los falsos positivos (FP) corresponden al número de registros Holter cuyo diagnóstico matemático indica anormalidad, mientras que la evaluación clínica es de normalidad. Los falsos negativos (FN) son el número de registros Holter evaluado por el experto clínico como normales y cuyos valores matemáticos indican anormalidad. Finalmente, los verdaderos negativos (VN) corresponden al número de registros Holter evaluados por el cardiólogo como normales y cuyos valores matemáticos indican normalidad.

Con el fin de establecer la concordancia entre el diagnóstico matemático y el clínico convencional se calculó el coeficiente Kappa, mediante la siguiente ecuación:

$$
K=\frac{C o-C a}{T o-C a}
$$

\section{Donde:}

Co: corresponde al número de concordancias observadas, (el número de casos clasificados igualmente, mediante la metodología matemática y los parámetros clínicos convencionales). To: corresponde a la totalidad de observaciones (el total de casos tanto normales como patológicos). Ca: corresponde a las concordancias atribuidas al azar, calculadas mediante la siguiente ecuación:

$$
C a=\left[\frac{\left(f_{1} C_{1}\right)}{T o}+\frac{\left(f_{2} C_{2}\right)}{T o}\right]
$$

Donde 11 corresponde al número de casos con valores matemáticos indicativos de normalidad; $\mathrm{C} 1$ representa el número de casos diagnosticados clínicamente como normales; f2 representa el número de casos diagnosticados como patológicos de acuerdo con los valores matemáticos; $\mathrm{C} 2$ es el número de casos diagnosticados clínicamente como patológicos y To representa la totalidad de casos.

\section{Resultados}

El diagnóstico clínico de 10 de los 80 registros Holter evaluados durante 21 y 19 horas seleccionados para el

\begin{tabular}{|c|c|}
\hline No. & Datos clínicos \\
\hline 1 & Bradicardia con presencia de vértigo y disnea \\
\hline 2 & Estudio dentro de los límites normales \\
\hline 3 & Arritmias por severo compromiso de la función \\
\hline 4 & Bradicardia \\
\hline 5 & Síncope en estudio \\
\hline 6 & Estudio dentro de los límites normales \\
\hline 7 & Infarto agudo de miocardio \\
\hline 8 & Infarto agudo de miocardio \\
\hline 9 & Dolor torácico taquiarritmia paroxística \\
\hline 10 & Arritmia cardiaca \\
\hline
\end{tabular}
estudio se puede ver en la Tabla 1 . Los valores de la frecuencia cardiaca para los registros Holter evaluados en 21 horas variaron entre 30 y $160 \mathrm{Lat} / \mathrm{min}$, siendo en total 27 rangos (Tabla 2). Los valores de la probabilidad que corresponden a los rangos evaluados en 21 horas variaron entre 0 y 0,489 (Tabla 2). El número total de latidos por hora tomó valores contenidos dentro de 20 rangos, entre 2000 y 6750 Lat/hora (Tabla 3). Las probabilidades halladas para el número total de latidos por hora variaron entre 0 y 0,524 . Los valores de la probabilidad correspondientes a los rangos evaluados en 19 horas variaron entre 0 y 0.472 . Los registros Holter evaluados en 19 horas variaron entre 45 y 190 Lat/min con un total de 30 rangos (Tabla 3). El número total de latidos por hora tomó valores contenidos dentro de 30 rangos, entre 2000 y 9250 Lat/hora. Las probabilidades halladas para el número total de latidos por hora variaron entre 0 y 0,526 (Tabla 3) Entre los casos normales, se encontraron dos casos con probabilidad menor o igual a 0,217 y ocho con probabilidad mayor o igual a 0,304; se evidenció que ningún caso de enfermedad aguda presentó valores con probabilidad menor o igual a 0,217 , mientras que todos presentaron valores mayores o iguales a 0,304 , tanto para los registros Holter evaluados en 21 como en 19 horas (Tabla 3).

En cuanto a los pacientes evaluados con los parámetros diagnósticos se encontró que la dinámica cardíaca de los pacientes 3 y 10 presentan dos estados en el tiempo. El paciente No 3, en 19 horas tiene un diagnóstico matemático de enfermedad (E), en cambio en 21 horas el estado es de evolución a la enfermedad (V). El paciente No 10 tiene un diagnóstico matemático de evolución a la enfermedad (V), y en 21 horas el estado es de enfermo (E). Estos resultados muestran que sin importar si hay un 
Tabla 2. Distribución de los valores de la probabilidad calculados a partir de los valores de la frecuencia cardiaca durante 21 y 19 horas, para los 10 registros Holter de la Tabla 1. En la primera columna se acotaron las frecuencias cardíacas en rangos, que acorde a la metodología, son de 5 latidos por minuto, pero se exhiben rangos de mayor tamaño para agrupar mayores cantidades de valores de probabilidad. Para cada registro Holter se anotaron los valores de probabilidad para cada rango indicando el máximo valor de probabilidad obtenido. Se señala en gris el primer registro Holter para cada hora. RFC: rango de frecuencias cardíacas.

\begin{tabular}{|c|c|c|c|c|c|c|c|c|c|c|c|c|c|c|c|c|c|c|c|c|}
\hline \multicolumn{11}{|c|}{21 horas } & \multicolumn{10}{|c|}{19 horas } \\
\hline RFC & 1 & 2 & 3 & 4 & 5 & 6 & 7 & 8 & 9 & 10 & 1 & 2 & 3 & 4 & 5 & 6 & 7 & 8 & 9 & 10 \\
\hline $\begin{array}{l}30 \\
40\end{array}$ & 0 & 0 & 0 & 0 & 0 & 0 & 0 & 0 & 0 & 0 & 0 & 0 & 0 & 0 & 0 & 0 & 0 & 0 & 0 & 0 \\
\hline $\begin{array}{l}45 \\
55\end{array}$ & $\begin{array}{r}0,044- \\
0,489\end{array}$ & $\begin{array}{r}0,014- \\
0,042\end{array}$ & 0,03 & $\begin{array}{lr}3 & 0 \\
0,167\end{array}$ & $\begin{array}{r}0,102- \\
0,184\end{array}$ & $\begin{array}{r}0- \\
0,042\end{array}$ & 0 & $\begin{array}{r}0,070- \\
0,085\end{array}$ & 5 & $\begin{array}{r}0- \\
0,167\end{array}$ & $\begin{array}{r}0,056- \\
0,472\end{array}$ & 0,054 & $\begin{array}{r}0,035- \\
0,228\end{array}$ & $\begin{array}{rr}- & 0- \\
3 & 0,185\end{array}$ & 0,119 & 0 & 0 & $\begin{array}{r}0- \\
0,089\end{array}$ & - $0-0,07$ & $\begin{array}{r}0 \\
7 \\
0,193\end{array}$ \\
\hline $\begin{array}{l}60 \\
70\end{array}$ & $\begin{array}{r}0- \\
0,200\end{array}$ & $\begin{array}{r}0,042- \\
0,056\end{array}$ & $\begin{array}{r}0,045- \\
0,136\end{array}$ & $\begin{array}{rr}- & 0,106- \\
6 & 0,227\end{array}$ & $\begin{array}{r}0,020- \\
0,122\end{array}$ & $\begin{array}{r}0,014- \\
0,042\end{array}$ & 0 & $\begin{array}{r}0,085- \\
0,127\end{array}$ & $\begin{array}{rr}- & 0,111- \\
7 & 0,403\end{array}$ & $\begin{array}{c}0,121- \\
0,167\end{array}$ & $\begin{array}{r}0- \\
0,194\end{array}$ & 0,054 & $\begin{array}{r}0,035- \\
0,158\end{array}$ & $\begin{array}{rr}- & 0,111- \\
3 & 0,241\end{array}$ & 0,024 & $\begin{array}{r}0,018- \\
0,054\end{array}$ & & $\begin{array}{r}0,054- \\
0,107\end{array}$ & $\begin{array}{rr}- & 0,105- \\
7 & 0,368\end{array}$ & $\begin{array}{cc}-0,123 \\
8 & 0,158\end{array}$ \\
\hline $\begin{array}{l}75 \\
85\end{array}$ & $\begin{array}{r}0- \\
0,044\end{array}$ & $\begin{array}{r}0,014- \\
0,028\end{array}$ & $\begin{array}{r}0,015- \\
0,030\end{array}$ & $\begin{array}{rr}-5,015- \\
0 & 0,076\end{array}$ & $\begin{array}{r}0,102- \\
0,143\end{array}$ & $\begin{array}{r}0,028- \\
0,085\end{array}$ & $\begin{array}{r}0,127- \\
0,211\end{array}$ & $\begin{array}{r}-0,085- \\
1 \quad 0,127\end{array}$ & $\begin{array}{rr}- & 0,014- \\
7 & 0,056\end{array}$ & $\begin{array}{r}0,030- \\
0,045\end{array}$ & $\begin{array}{r}0- \\
0,056\end{array}$ & 0,054 & $\begin{array}{r}0- \\
0,018\end{array}$ & $0-0,13$ & 0,095 & 0,054 & $\begin{array}{r}0- \\
0,089\end{array}$ & $\begin{array}{r}-0,036- \\
0,143\end{array}$ & $\begin{array}{rr}- & 0,018- \\
3 & 0,053\end{array}$ & $\begin{array}{cc}- & 0,035 \\
3 & 0,053\end{array}$ \\
\hline $\begin{array}{r}90 \\
100\end{array}$ & 0 & 0 & $\begin{array}{l}0,045- \\
0,121\end{array}$ & $\begin{array}{r}-0,015- \\
0,076\end{array}$ & 0,02 & $\begin{array}{l}0,042- \\
0,099\end{array}$ & $\begin{array}{l}0,085- \\
0,141\end{array}$ & $\begin{array}{r}-0,042- \\
0,056\end{array}$ & $\begin{array}{l}0- \\
0,014\end{array}$ & $\begin{array}{l}0,015- \\
0,045\end{array}$ & 0 & 0,018 & $\begin{array}{l}0,07- \\
0,14\end{array}$ & $\begin{array}{l}0- \\
0,093\end{array}$ & 0,024 & $\begin{array}{l}0,071- \\
0,089\end{array}$ & $\begin{array}{r}-0,071- \\
0,196\end{array}$ & $\begin{array}{r}-0,071- \\
0,125\end{array}$ & $\begin{array}{l}-0- \\
0,018\end{array}$ & $\begin{array}{l}0,018 \\
0,035\end{array}$ \\
\hline $\begin{array}{l}105 \\
125\end{array}$ & $\begin{array}{r}0- \\
0,022\end{array}$ & 0,014 & $\begin{array}{r}0- \\
0,045\end{array}$ & $\begin{array}{rr}- & 0- \\
5 & 0,015\end{array}$ & 0 & $\begin{array}{r}0,014- \\
0,07\end{array}$ & $\begin{array}{r}0- \\
0,155\end{array}$ & 5 & 0 & $\begin{array}{r}0- \\
0,076\end{array}$ & 0 & 0 & 0,035 & 0 & 0 & $\begin{array}{r}0,018- \\
0,089\end{array}$ & $\begin{array}{rr}- & 0- \\
9 & 0,214\end{array}$ & $\begin{array}{rr}0 \\
-\quad 0,036\end{array}$ & $\begin{array}{ll}- & 0\end{array}$ & $0 \quad 0-0,07$ \\
\hline $\begin{array}{l}130 \\
150\end{array}$ & 0 & 0,085 & 0 & 0 & 0 & $\begin{array}{r}0,014- \\
0,085\end{array}$ & 0 & 0 & 0 & $\begin{array}{r}0- \\
0,015\end{array}$ & 0 & 0 & 0 & 0 & 0 & $\begin{array}{r}0,018- \\
0,089\end{array}$ & 0 & 0 & 0 & $\begin{array}{r}0 \\
0,018\end{array}$ \\
\hline$>155$ & 0 & 0 & 0 & 0 & 0 & 0 & 0 & 0 & 0 & 0 & 0 & 0 & 0 & 0 & 0 & 0 & 0 & 0 & 0 & 0 \\
\hline Máximo & 0,489 & 0,085 & 0,197 & $\begin{array}{ll}7 & 0,227\end{array}$ & 0,184 & 0,099 & 0,211 & $1 \quad 0,127$ & $\begin{array}{ll}7 & 0,403\end{array}$ & 0,167 & 0,472 & 0,089 & 0,228 & $\begin{array}{ll}3 & 0,241\end{array}$ & 0,214 & 0,089 & 0,214 & $4 \quad 0,143$ & $\begin{array}{ll}3 & 0,368\end{array}$ & 0,193 \\
\hline
\end{tabular}

Tabla 3. Distribución de los valores de la probabilidad calculados a partir de la cantidad de latidos cardiacos durante 21 y 19 horas, para los 10 registros Holter de la Tabla 1. En la primera columna se acotaron las frecuencias cardíacas en rangos, que acorde a la metodología, son de 250 latidos por minuto, pero se exhiben rangos de mayor tamaño para agrupar mayores cantidades de valores de probabilidad. Se señala en gris el primer registro Holter para cada hora RL: rangos de latidos.

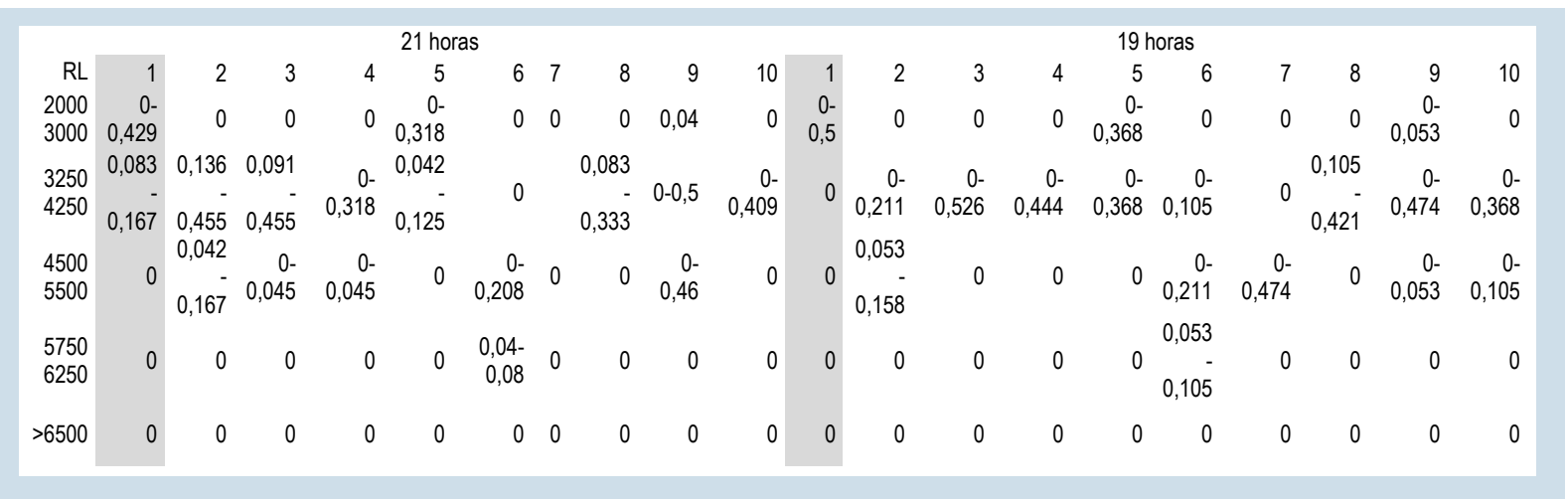

estado de evolución a la enfermedad o enfermedad, la dinámica cardíaca durante 21 horas se encuentra atrapada dentro de los rangos matemáticos que definen la enfermedad (ver Tabla 4). También, se evidencia la capacidad del método de establecer evolución de las patologías cardíacas, lo cual sería útil en el seguimiento de pacientes. Se obtuvieron valores de sensibilidad y especificidad del $100 \%$ y el coeficiente Kappa fue de 1.

\section{Discusión:}

Este es el primer trabajo que evidencia la capacidad diag- nóstica de una metodología fundamentada en la teoría de la probabilidad y los sistemas dinámicos durante 19 horas, en un marco de un estudio ciego con 80 casos. Se corrobora su utilidad diagnóstica, alcanzando los mayores valores de sensibilidad y especificidad, y un coeficiente Kappa igual a 1. Se trata de un diagnóstico matemático útil en cualquier caso particular pues se fundamenta en el orden matemático que subyace al fenómeno y no en parámetros poblacionales. Los parámetros matemáticos diferencian completamente estados cardíacos normales de anormales, siendo de utilidad para determinar la efec- 
Tabla 4. Diagnóstico matemático realizado a partir de los rangos de la frecuencia cardiaca registrada durante 21 y 19 horas para los 10 registros Holter de la Tabla 1. Se exhiben el número de rangos de frecuencias cardíacas (R), los valores de las sumatorias de probabilidad (S) y a continuación los subparámetros diferenciadores de las dinámicas cardíacas como la diferencia entre los rangos de los dos valores de frecuencia con probabilidad más elevada (D), el valor máximo de la probabilidad calculado y si esta es $\leq 0,217$ o $\geq 0,304$ con lo cual finalmente se llega al diagnóstico matemático (DxM).

No. Número de caso, R: número de rangos; S: sumatoria de probabilidad; D: diferencia entre los rangos de los dos valores de frecuencia con probabilidad más elevada; Máx P: valor máximo de la probabilidad calculado; DxM: diagnóstico matemático; E: enfermedad; N: normal; V: evolución a la enfermedad.

\begin{tabular}{|c|c|c|c|c|c|c|c|c|c|c|c|c|c|c|c|c|}
\hline \multirow{3}{*}{ No. } & \multicolumn{8}{|c|}{21 horas } & \multicolumn{8}{|c|}{19 horas } \\
\hline & \multirow{2}{*}{$\mathbf{R}$} & \multirow{2}{*}{ S } & \multirow{2}{*}{$>0,319$} & \multirow{2}{*}{ D } & \multirow{2}{*}{ Máx $\mathrm{P}$} & \multicolumn{2}{|c|}{ P. Lat } & \multirow{2}{*}{ DxM } & \multirow{2}{*}{ DxM } & \multicolumn{2}{|c|}{ P. Lat } & \multirow{2}{*}{ Máx P } & \multirow{2}{*}{ D } & \multirow{2}{*}{$>0,319$} & \multirow{2}{*}{$S$} & \multirow{2}{*}{$\mathbf{R}$} \\
\hline & & & & & & $<=0,217$ & $>=0,304$ & & & $>=0,304$ & $<=0,217$ & & & & & \\
\hline 1 & 13 & 0,333 & $x$ & 10 & 0,455 & & $x$ & $\mathrm{E}$ & $E$ & $x$ & & 0,5 & 15 & $X$ & 0,667 & 6 \\
\hline 2 & 22 & 0,155 & & 5 & 0,292 & $X$ & & $\mathrm{~N}$ & $\mathrm{~N}$ & & $X$ & 0,211 & 10 & & 0,161 & 20 \\
\hline 3 & 16 & 0,288 & & 10 & 0,409 & & $X$ & V & V & $X$ & & 0,526 & 10 & $X$ & 0,386 & 12 \\
\hline 4 & 7 & 0,689 & $X$ & 15 & 0,524 & & $X$ & $E$ & $E$ & $X$ & & 0,444 & 5 & $x$ & 0,426 & 7 \\
\hline 5 & 8 & 0,708 & & 5 & 0,227 & & $X$ & $E$ & $E$ & $X$ & & 0,368 & 25 & $X$ & 0,381 & 10 \\
\hline 6 & 20 & 0,197 & & 5 & 0,25 & $X$ & & $\mathrm{~N}$ & $\mathrm{~N}$ & & $x$ & 0,211 & 5 & & 0,179 & 17 \\
\hline 7 & 7 & 0,38 & $x$ & 5 & 0,48 & & $X$ & $E$ & $E$ & $X$ & & 0,474 & 15 & $x$ & 0,411 & 7 \\
\hline 8 & 12 & 0,254 & & 10 & 0,333 & & $X$ & $E$ & $E$ & $X$ & & 0,421 & 10 & & 0,268 & 12 \\
\hline 9 & 10 & 0,397 & $x$ & 5 & 0,455 & & $X$ & $E$ & $E$ & $X$ & & 0,474 & 5 & $x$ & 0,684 & 8 \\
\hline 10 & 10 & 0,347 & $X$ & 35 & 0,318 & & $X$ & $E$ & $E$ & $x$ & & 0,368 & 10 & $X$ & 0,351 & 16 \\
\hline
\end{tabular}

tividad de tratamientos, así como la evolución del paciente en rangos menores de tiempo. Adicionalmente, al analizar diferentes parámetros tanto respecto a la frecuencia cardíaca como al número de latidos, permite evidenciar alteraciones leves potencialmente significativas, no detectadas mediante parámetros convencionales.

Esta metodología, basada en las leyes de la probabilidad, se consolida como una forma de evaluar los patrones cardíacos obtenidos en el Holter sin emplear un número elevado de parámetros. Su mayor utilidad radica en que podría ser incorporada automáticamente en dispositivos electrónicos de electrocardiografía continua, aprovechando su carácter puramente matemático para obtener diagnósticos de elevada precisión ${ }^{14}$, sin depender de factores epidemiológicos. Su incorporación al ámbito clínico sería útil, tanto en el diagnóstico, como en el seguimiento continuo de pacientes a nivel ambulatorio y hospitalario, tanto en las Unidades de Cuidado Intensivo, como en las alas hospitalarias estándar. Sin embargo, se requieren estudios con mayores cantidades de pacientes para confirmar su capacidad diagnóstica.

Cabe destacar que el método matemático arroja que un número de latidos inferior a 3.000 o superior a 6.250 es indicativo de evolución hacia la enfermedad. Ello sugeriría su utilidad en casos de patologías subdiagnosticadas o en las cuales los pacientes aún no exhiban síntoma. Ello orientaría a indicar seguimiento del paciente; incluso, el método podría emplearse para evaluar la respuesta de los pacientes a las intervenciones terapéuticas.
Una rama de la cardiología ha centrado sus esfuerzos en el estudio de la variabilidad de la frecuencia cardíaca con el propósito de indicar diagnósticos y predicciones de interés clínico ${ }^{10,12,18,19}$; Por ejemplo, bajos valores de los parámetros como el SDNN, del que se parte como Gold Standard para estratificar el riesgo cardíaco en mediciones de 24 horas, parecen asociarse a mortalidad en sepsis $^{20}$ y a mayor mortalidad en la Unidad de Cuidados Intensivos ${ }^{21}$ si sus valores se encuentran menores a $50^{22}$. Sin embargo, hasta el momento esto no se ha demostrado rigurosamente como cierto para este parámetro ni para otros como pNN50, NN, sNN50, ${ }^{23}$. Siendo así, esta perspectiva no permite el establecimiento de diferencias y diagnósticos precisos en cada caso particular y más aún, que requieren de valores normalizados locales para cada parámetro, necesitando de estudios poblaciones significativos para caracterizar y validar cada parámetro. En este trabajo se presenta un método cuantitativo que permite diferenciar dinámicas cardíacas normales de anormales con base en la autoorganización matemática intrínseca del sistema, independiente de factores de riesgo y otros epidemiológicos locales.

Desde esta perspectiva acausal, se han desarrollado otras metodologías diagnósticas predictivas. Tal es el caso de una metodología basada en la ocupación de los atractores cardíacos caóticos en el espacio fractal de Box- Counting, que tiene la capacidad de diferenciar entre estados agudos, crónicos y normales, incluso en pacientes cursando con infarto agudo de miocardio, alcanzando va- 
lores de sensibilidad y especificidad del $100 \%{ }^{13}$. Continuando con esta línea de investigación, se ha desarrollado otras metodologías fundamentadas en la probabilidad, la entropía y las proporciones de la entropía, que permite hacer diagnósticos certeros $^{24}$ y cuya utilidad fue corroborada en diferentes poblaciones, incluyendo pacientes de la UCI. En el caso de un paciente con una estancia de 15 días en la Unidad de Cuidado Intensivo, se predijo mediante este método la evolución hacia el deterioro, aun cuando clínicamente no se evidenciaba lo anterior, mostrando la utilidad de las metodologías físicas y matemáticas, y su capacidad de evaluar cuantitativamente los sistemas cardíacos ${ }^{25}$.

Otros estudios en medicina realizados en los marcos de las observaciones anteriores han permitido realizar predicciones y establecer diagnósticos en la morfometría arterial $^{26}$ y celular ${ }^{27}$, la hematología 28 , o la infectología, en la predicción del número de linfocitos TCD4 en pacientes cursando con infección por el $\mathrm{VIH}^{29}$. También, se han realizado predicciones de mortalidad en la $\mathrm{UCI}^{30}$. Estas investigaciones evidencian la importancia de la implementación de metodologías matemáticas en la medicina.

\section{Agradecimientos:}

Este artículo es resultado del proyecto INV 1950 financiado por la Universidad Cooperativa de Colombia, sede Bogotá. Agradecemos a los Doctores Fernando Colmenares, Director de Investigaciones; Leonardo Galindo, Director DINAI Nacional; Andrés Mena, Director DINAI Bogotá; Eva Prada, Directora de Sede Bogotá; y Edgar López, Decano de la Facultad de Ingeniería, por su apoyo a nuestras investigaciones.

Extendemos nuestro agradecimiento al Centro de Investigaciones de la Clínica del Country, a la Doctora Adriana Lizbeth Ortiz, epidemióloga, y Silvia Ortiz, enfermera jefe, y a los Doctores Tito Tulio Roa, Director de Educación Médica; Jorge Alberto Ospina, Director Médico; y Alfonso Correa, Director del Centro de Investigaciones, por el apoyo a nuestro grupo de investigación.

\section{Descargos de Responsabilidad}

El presente resultado hace parte de los productos del proyecto INV 1950 financiado por la Universidad Cooperativa de Colombia.

\section{Dedicatoria}

A nuestros hijos.

\section{Referencias}

1 PEITGEN H, JURGENS H, SAUPE D. Chaos and fractals; new frontiers of science. New York: Springer; 1992.

2 PEITGEN H, JURGENS H, SAUPE D. Strange attractors, the locus of chaos. En: Chaos and Fractals: New Frontiers of Science. Springer-Verlag. New York; 1992. pp. 655-768.

3 DEVANEY R. A first course in chaotic dynamical systems theory and experiments. Reading Mass.: Addison- Wesley 1992.

4 DENTON T, DIAMOND G, HELFANT R, KHAN S, KARAGUEUZIAN H. Fascinating rhythm: A primer on chaos theory and its application to cardiology. Am Heart J 1990; 6(1): 14191440 .

5 WEST BJ. Fractal physiology and chaos medicine. London. World Scientific Publishing Co. 1990.
6 GOLDBERGER A. Non-linear dynamics for clinicians: chaos theory, fractals, and complexity at the bedside. Lancet 1996; 347: 1312-1314.

7 GOLDBERGER AL, RIGNEY DR, WEST BJ. Chaos and fractals in human physiology. Sci Am 1990; 262: 42-49.

8 OMS- Centro de prensa. Enfermedades Cardiovasculares. Nota informativa Enero de 2011. Disponible en http://www.who.int/ mediacentre/factsheets/fs317/es/index.html. Acceso: Abril de 2012.

9 PAN AMERICAN HEALTH ORGANIZATION. All rights reserved. Las enfermedades no transmisibles (ENT), nuestro reto. Disponible en: https://www.paho.org/col/index.php?option=com_content $\&$ view $=$ article $\&$ id $=1756$ : las-enfermedades-no-transmisibles-ent-nuestro-reto\&Itemid $=487$ 
10 BARRET PM, KOMATIREDDY R, HAASER S, TOPOL S, SHEARD J, ENCINAS J, et al. Comparison of 24-hour Holter Monitoring with 14-day Novel Adhesive Patch Electrocardiographic Monitoring. Am J Med. 2014:127(1): 10.1016/j.amjmed.2013.10.003.

11 GOLDBERGER AL. Fractal dynamics in physiology: Alterations with disease and angin. PNAS 2002; 99: 2466-2472.

12 HUIKURI H, MÄKIKALLO T, PENG CH, GOLDBERGER A, HINTZE U, MOLLER M. Fractal correlation properties of $\mathrm{R}-\mathrm{R}$ interval dynamics and mortality in patients with depressed left ventricular function after an acute myocardial infarction. Circulation 2000; 101: 47

13 RODRÍGUEZ J, PRIETO S, CORREA C, DOMÍNGUEZ D, PARDO JM, MENDOZA F, et al. Clinical application of a cardiac diagnostic method based on dynamic systems theory. Res J Cardiol 2017; 10(Issue 1):1-7.

14 RODRÍGUEZ J, CORREA C, ORTIZ L, PRIETO S, BERNAL P, AYALA J. Evaluación matemática de la dinámica cardiaca con la teoría de la probabilidad. Rev Mex Cardiol. 2009; 20 (4):183-9.

15 RODRÍGUEZ J, CORREA C, PRIETO S, BERNAL P, FORERO G, SALAZAR G, et al. Confirmación del método de ayuda diagnóstica de la dinámica cardiaca de aplicación clínica desarrollado con base en la teoría de la probabilidad. Rev MED 2011; 19 (2): 167-178.

16 RODRÍGUEZ J, PRIETO P, MENDOZA F, PINILLA L, CORREA C, SORACIPA Y, et al. Evaluación probabilista de la dinámica cardiaca arrítmica con y sin metoprolol. CES Med 2017; 31(2):144-154.

17 BODAPATI RK, KIZER JR, KOP WJ, KAMEL H, STEIN PK. Addition of 24-Hour Heart Rate Variability Parameters to the Cardiovascular Health Study Stroke Risk Score and Prediction of Incident Stroke: The Cardiovascular Health Study. JACC Heart failure. 2017;5(6):423-431. doi:10.1016/j. jchf.2016.12.015.

18 ERNST G. Hidden Signals-The History and Methods of Heart Rate Variability. Frontiers in Public Health. 2017;5:265. doi:10.3389/fpubh.2017.00265.

19 WALLÉN MB, HASSON D, THEORELL T, CANLON B, OSIKA W. Possibilities and limitations of the Polar RS800 in measuring heart rate variability at rest. Eur J Appl Physiol. 2012;112(3):1153-65. doi: 10.1007/s00421-011-2079-9.

20 De CASTILLHO FM, RIBEIRO ALP, NOBRE V, BARROS G, De SOUSA MR. Heart rate variability as predictor of mortality in sepsis: A systematic review. PLoS ONE. 2018;13(9): e0203487. DOI: 10.1371/journal.pone.0203487.
21 MORIDANI MK, SETAREHDAN SK, NASRABADI AM, HAJINASROLLAH E. Analysis of heart rate variability as a predictor of mortality in cardiovascular patients of intensive care unit. Biocybernetics and Biomedical Engineering. 2015;35(4):217-226. DOI: 10.1016/j.bbe.2015.05.004

22 SHAFFER F, GINSBERG JP. An Overview of Heart Rate Variability Metrics and Norms. Fron Public Health. 2017;5:258. DOI: $10.3389 /$ fpubh.2017.00258.

23 WU L, JIANG Z, LI C, SHU M. Prediction of heart rate variability on cardiac sudden death in heart failure patients: A systematic review. Int J Cardiol. 2015;174(3):857-860. DOI: 10.1016/j.ijcard.2014.04.176

24 RODRÍGUEZ J. Entropía proporcional de los sistemas dinámicos cardiacos: Predicciones físicas y matemáticas de la dinámica cardiaca de aplicación clínica. Rev Colomb Cardiol. 2010; 17:115-129.

25 RODRÍGUEZ J, PRIETO S, BERNAL P, IZASA D, SALAZAR G, CORREA C, et al. Entropía proporcional aplicada a la evolución de la dinámica cardiaca. Predicciones de aplicación clínica. En: Rodríguez LG, Coordinador. La emergencia de los enfoques de la complejidad en América Latina: desafíos, contribuciones y compromisos para abordar los problemas complejos del siglo XXI. Tomo 1,1a ed. Buenos Aires: Comunidad Editora Latinoamericana; 2015. p. 315-44.

26 RODRÍGUEZ J, PRIETO S, CORREA C, BERNAL P, PUERTA G, VITERY S, et al. Theoretical generalization of normal and sick coronary arteries with fractal dimensions and the arterial intrinsic mathematical harmony. BMC Medical Physics. 2010;10:1-6.

27 RODRÍGUEZ J, PRIETO S, CORREA C, DOMINGUEZ D, CARDONA DM, MELO M. Geometrical nuclear diagnosis and total paths of cervix cell evolution from normality to cancer. J Can Res Ther 2015; 11(1): 98-104.

28 CORREA C, RODRÍGUEZ J, PRIETO S, ÁLVAREZ L, OSPINO B, MUNÉVAR A, et al. Geometric diagnosis of erythrocyte morphophysiology: Geometric diagnosis of erythrocyte. J. Med. Med. Sci. 2012; 3(11): 715-720.

29 RODRÍGUEZ J, PRIETO S, CORREA C, PÉREZ C, MORA J, BRAVO J, et al. Predictions of CD4 lymphocytes' count in HIV patients from complete blood count. BMC Medical Physics. BMC Medical Physics. 2013;13:3.

30 RODRÍGUEZ J. Dynamical systems applied to dynamic variables of patients from the Intensive Care Unit (ICU). Physical and mathematical Mortality predictions on ICU. J.Med.Med. Sci. 2015; 6(8): 102-108. 